\title{
Modelagem computacional de efeitos de distorções não lineares para guitarra elétrica
}

\author{
Thomaz Chaves A. Oliveira ${ }^{1}$ \\ Gilmar Barreto ${ }^{1}$ \\ Alexander Mattioli Pasqual ${ }^{2}$
}

\begin{abstract}
Resumo:
Este trabalho tem como objetivo revisar e simular alguns dos métodos de emulação computacional de efeitos de distorção de guitarras elétricas e de amplificadores valvulados que têm caracterizado os timbres desse instrumento nos últimos cinquenta anos. Recentemente, devido à disseminação do processamento digital de sinais, essas distorções têm sido reproduzidas por meio de softwares embarcados ou na forma de pluggins em softwares de estúdio. Para a simulação de distorções, duas abordagens basicamente são utilizadas: caixa preta e caixa branca. Na primeira, waveshapers estáticos simplificam o sistema com equações não lineares que aproximam o comportamento do aparelho. Algumas das equações presentes na literatura são simuladas neste trabalho, com as respectivas respostas dos sistemas não lineares no tempo e na frequência. Na abordagem caixa branca, os parâmetros dos circuitos são levados em conta, e a modelagem pode ser realizada usando os Wave Digital Filters ou sistemas de equações diferenciais ordinárias em sua representação no espaço de estados, os quais são solucionados por métodos numéricos. As simulações caixa branca apresentam resultados mais precisos, porém demandam maiores recursos computacionais, sendo necessário um compromisso entre precisão e eficiência para a simulação em tempo real.
\end{abstract}

Palavras-chave: Modelos digitais virtualmente analógicos. Processamento digital de sinais de áudio. Modelos de eletrônica analógica. Guitarra elétrica. Computação aplicada.

\begin{abstract}
This work objective is to review and to simulate some of the guitar distortion algorithms, either simulating tube amplifiers or distortion pedals that have characterized the timbral characteristics of these instruments over the last 50 years. In recent times, the dissemination of digital signal processing has enabled the simulation of such devices either by embedded systems or by studio recording software plugins. There are basically two approaches for the simulation of these devices, a black box and a white box. In the black box approach, static waveshapers simplify the system by approximating its response by a nonlinear function that resembles the devices operation. Some of the equations presented in the literature are simulated in this work, and the resulting output signals in the time and frequency domains are given. In the white box approach, circuit parameters are used in modeling the physical system. These models can either be simulated by Wave Digital Filters or by a system of ordinary differential equations in the state space representation, which is solved by numerical methods. White box approaches yield more precise results, but demand more computer resources, therefore a compromise between efficiency and precision must be found for real time simulation.
\end{abstract}

Keywords: Virtual analog digital models. Digital audio signal processing. Analog electronic models. Electric guitar. Applied computing.

\footnotetext{
${ }^{1}$ Departamento de Semicondutores Fotônica e Instrumentação - Faculdade de Engenharia Elétrica e de Computação Universidade Estadual de Campinas

\{thomazchaves@gmail.com, gbarreto@dmcsi.fee.unicamp.br\}

${ }^{2}$ Departamento de Engenharia Mecânica - Universidade Federal de Minas Gerais

\{ampasqual@gmail.com\}
}

http://dx.doi.org/10.5335/rbca.2013.2877 


\section{Introdução}

Atualmente, discute-se muito sobre a interseção entre os domínios da eletrônica e da música com o objetivo de ampliar o ferramental de criação disponível aos compositores, instrumentistas profissionais e amadores, arranjadores e produtores musicais. O debate acerca da junção entre computação e música tem se intensificado desde a segunda metade da década de 1970. Os computadores digitais, a partir de sua popularização, tornaram-se qualquer tipo de aplicação sonora. Nesse contexto, a tecnologia digital tem evoluído no sentido de proporcionar (por meio de simulações computacionais cada vez mais precisas) a virtualização de ambientes de ensino, ambientes acústicos, instrumentos musicais, efeitos analógicos, entre outros. Este trabalho enquadra-se no ramo dos efeitos eletrônicos analógicos virtuais e, mais especificamente, refere-se a amplificadores valvulados e efeitos analógicos de distorção não lineares, os quais geram características sonoras peculiares e demandam altos esforços computacionais para uma simulação precisa.

Essas simulações podem ser consideradas, contextualmente, ferramentas em potencial para referenciar timbres clássicos de certos músicos da guitarra elétrica, tratando-se de ferramentas com potencial educativo. À medida que a modelagem computacional dos circuitos se tornar mais precisa, melhor será a reprodução virtual do timbre associado a um determinado guitarrista.

Para a compreensão do contexto deste trabalho, inicialmente, deve-se entender como a distorção de pedais ou de amplificadores para guitarra elétrica é utilizada musicalmente. Quando se tornaram populares, nas décadas de 1950 e 60, os amplificadores para instrumentos elétricos utilizavam válvulas termiônicas em seus circuitos, tal como o amplificador mostrado na Figura 1-b. Os amplificadores valvulados, quando operam além da sua capacidade máxima de amplificação (regime de overdrive), promovem distorções não lineares no sinal, reforçando o conteúdo harmônico dos acordes e das notas de um modo peculiar. A distorção característica desses equipamentos tornou-se um atrativo para os músicos nas décadas de 1960 e 70 . À medida que a tecnologia evoluiu em direção a dispositivos de estado sólido, os novos amplificadores, utilizando transistores em vez de válvulas, foram incapazes de reproduzir as características sonoras dos amplificadores à válvula. Segundo Bussey [4], os músicos utilizam adjetivos como "vazio", "magro", ou mesmo "metálico" para descrever a sonoridade dos amplificadores transistorizados, enquanto "quente", "redondo" e "punchier" (expressão inglesa que descreve a resposta imediata do amplificador, uma analogia à intensidade de um "soco") são associados aos amplificadores valvulados. A partir do final da década de 1960, distorções adicionais tornaram-se uma necessidade dos guitarristas. Assim, diversos dispositivos transistorizados foram criados para gerar mais distorção no sinal da guitarra elétrica, tal como o pedal Fuzz Face mostrado na Figura 1-a. Os pedais podem ser valvulados, transistorizados, ou mais recentemente, digitais. A variedade de distorções possíveis de se obter em dispositivos analógicos e digitais permite expandir os horizontes da guitarra elétrica, indo de uma sútil distorção valvulada adequada ao Blues e ao Jazz, até uma distorção extrema adequada a alguns gêneros de Rock.

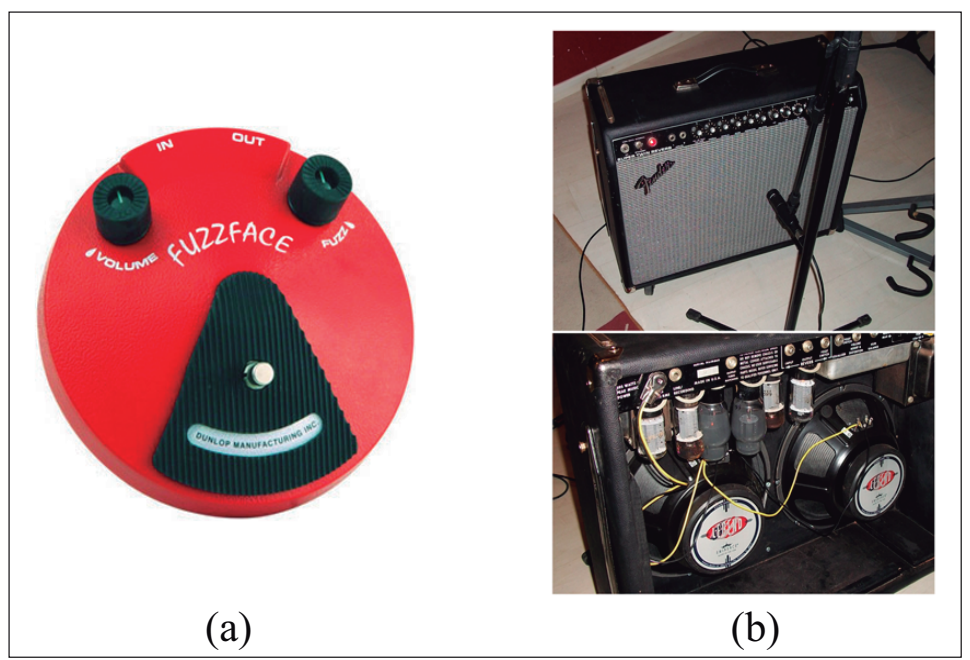

Figura 1: (a) Pedal de distorção transistorizado , (b) Amplificador valvulado. 
A partir do começo da década de 1990, a simulação dos amplificadores valvulados e outros dispositivos analógicos tomou o rumo do processamento digital de sinais ("Digital Signal Processing”- DSP). Uma das principais vantagens em utilizar algoritmos computacionais de simulação em vez de circuitos analógicos é a versatilidade: novos parâmetros podem ser carregados para a memória quando o usuário assim o desejar, de modo que um mesmo dispositivo pode simular a sonoridade de diferentes amplificadores analógicos, efeitos de distorção, entre outros [27]. Além disso, os amplificadores valvulados virtuais são mais baratos, mais leves e menos volumosos que os amplificadores valvulados reais. Segundo Pakarinen [32], à medida que os sistemas digitais vêm avançando, espera-se que amplificadores e pedais de distorções virtuais venham a substituir os pesados amplificadores valvulados. Essa tendência a simular equipamentos analógicos utilizando técnicas de processamento digital de sinais originou o termo "Virtual Analog" [29] ou virtualmente analógicos. Atualmente, devido à ampla disponibilidade de recursos computacionais e dos inúmeros circuitos analógicos com potencial para serem modelados, a simulação virtualmente analógica tem sido uma área de intensa pesquisa, tanto no meio acadêmico quanto nas empresas de desenvolvimento de pluggins de áudio para estúdio, com diversas patentes registradas.

Em razão das características dos amplificadores valvulados já descritas, a maioria dos esforços de modelagem de efeitos de distorções para guitarras recai sobre a modelagem computacional de amplificadores valvulados. Guitarristas sempre desejam que um amplificador virtual aproxime ao máximo a sonoridade do amplificador original que está sendo simulado. Um modelo digital de um amplificador valvulado deve ser flexível e realístico, de modo a recriar as características sonoras com a maior fidelidade possível. Os algoritmos que simulam estes amplificadores não podem ser computacionalmente ineficientes; devem ser simples o suficiente de maneira que possam ser executados em diversas instâncias paralelamente em uma mesma CPU na forma de pluggins [28]. Pakarinen e Yeh [32] apresentam um estudo preliminar das técnicas mais utilizadas para simular esse tipo de amplificador. No entanto, é inexistente um estudo mais amplo que também envolva as técnicas para a simulação de distorções de pedais e outros dispositivos; o presente trabalho propõe preencher essa lacuna. Neste trabalho, alguns dos métodos da literatura de simulação de efeitos de guitarra foram simulados, sendo implementadas no ambiente MATLAB, mediante utilização de um tom de excitação de $1 \mathrm{kHz}$ para cada função waveshaper não linear, a uma taxa de amostragem de $96 \mathrm{kHz}$. Essa frequência foi utilizada por ser altamente perceptível ao ouvido humano, tratando-se de um sinal muito empregado para testes de áudio. O sinal amostrado a $96 \mathrm{kHz}$ deve-se ao fato de suprimir o efeito de aliassing. A análise das distorções não lineares foi feita nos domínios do tempo e da frequência, com os resultados plotados em ambos os domínios.

\section{Modelagem de sistemas lineares e não lineares}

Sistemas físicos podem ser matematicamente modelados como sistemas lineares e não lineares. Em sistemas de amplificação, os circuitos lineares correspondem aos filtros (malhas de resistores e capacitores) conhecidos como tonestack. Um sistema linear não introduz frequências novas no sinal e é totalmente descrito por sua resposta ao impulso, desde que o sistema também seja invariante no tempo. Além disso, uma resposta linear segue o princípio das sobreposições de sinais [35], pois a resposta do sistema é conhecida, e o computador poderá recriá-la digitalmente na forma de um filtro digital.

Um sistema não linear, por sua vez, não pode ser caracterizado por sua resposta ao impulso e não obedece ao princípio da sobreposição. Um dispositivo amplificador de sinais é um sistema cuja relação entre a entrada e a saída não é linear. Dessa maneira, pode gerar distorção não linear, mesmo em pequenas doses no sinal de saída, gerando novas frequências ou harmônicos. Alguns exemplos de elementos de circuitos não lineares: diodos, transistores, amplificadores operacionais e válvulas termiônicas.

A Figura 2 ilustra as diferenças entre as distorções não lineares e lineares introduzidas por diferentes circuitos, onde o sinal indicado pelo número 1 é a entrada dos sistemas. Em (2) ocorre distorção linear por meio de um filtro passa-altas RC, em (3) ocorre distorção linear por um filtro passa baixas RC, em (4) ocorre baixa distorção não linear presente em circuitos valvulados, e em (5) ocorre alta distorção não linear típica em diodos grampeadores. 


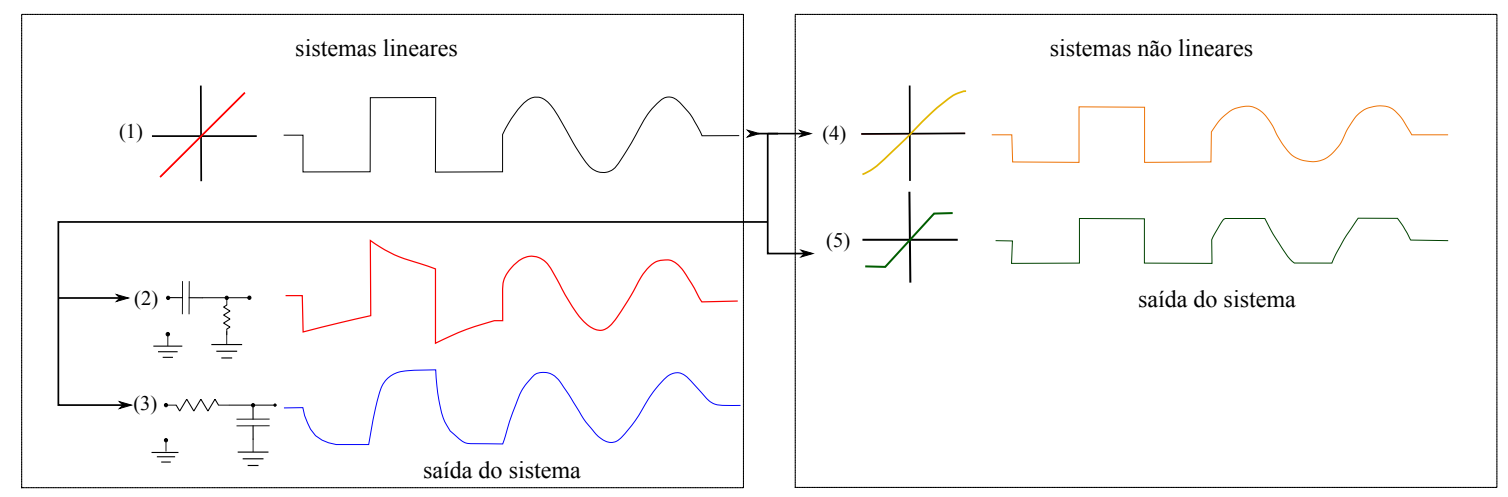

Figura 2: Distorções lineares e não lineares.

\section{Simulação de sistemas não lineares}

A distorção não linear, que muitas vezes está incluída propositalmente em gravações fonográficas ou em aparelhos analógicos ou digitais, pode ser modelada matematicamente como uma série temporal gerada por alguma função não linear [13]. Em sistemas analógicos e digitais esse tipo de distorção pode ocorrer quando: dispositivos ativos como transistores ou válvulas dão origem a distorções por ultrapassarem os limiares de amplificação conhecidos como regimes de overdrive; distorção de cruzamento (crossover) em amplificadores polarizados em classe $\mathrm{B}$ ou $\mathrm{AB}$ com baixa corrente quiescente; saturação de uma mídia analógica como uma fita; overflow do número de bits em uma gravação digital; sulco de um disco de vinil que possua danos, deformações ou mesmo flexibilidade indesejada gerando distorções no sinal a ser reproduzido.

No ramo de efeitos sonoros, muitas são as aplicações de algoritmos que processam o som de maneira não linear, ou que buscam replicar algum fenômeno físico não linear como os descritos anteriormente, sendo os mais comuns: algoritmos de processamento dinâmicos (compressores); simuladores de circuitos distorcedores como pedais e amplificadores; simulações de gravadoras de fita magnética e outros processamentos sonoros de realçamento baseados em psicoacústica. Esses processamentos adicionam componentes espectrais harmônicos ou não harmônicos que não estão presentes no sinal de entrada. Essas distorções são introduzidas por meio das características não lineares de transferências peculiares, como as de válvulas termiônicas. A aplicação musical desses dispositivos que geram distorções é uma arte e uma das principais ferramentas para músicos e engenheiros de som [9].

A distorção não linear que desejamos simular digitalmente é o overdrive. Um modelo digital, nesse caso, tem o objetivo de realizar um mapeamento de uma entrada sem distorção $\mathrm{s}[n]$ para uma saída distorcida $\mathrm{x}[n]$, como representado na Figura 3.

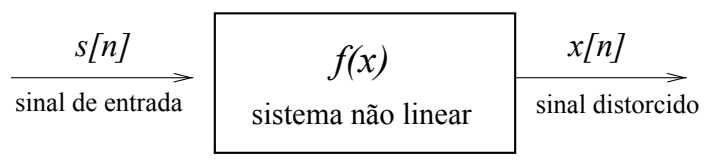

Figura 3: Representação de um sistema de distorção não linear.

\section{Alteração estática na forma de onda ou Waveshaping estático}

Modelos digitais de circuitos distorcedores podem ser implementados por meio de sistemas não lineares com ou sem memorização. Nos sistemas sem memorização, as variáveis não armazenam valores de estados anteriores do sistema. Por outro lado, em sistemas com memorização, as variáveis podem armazenar estados anteriores, como ocorre, por exemplo, em circuitos com capacitores ou indutores, cujas tensões e correntes dependem dos valores dessas variáveis em instantes anteriores. 
Os circuitos de interesse neste trabalho são categorizados como sistemas não lineares com memorização. Uma vez que todo e qualquer modelo é uma simplificação de sistemas físicos [16], busca-se utilizar um conjunto de equações que represente um sistema da melhor maneira possível. Um método para se aproximar o comportamento de um circuito de distorção não linear com memorização envolve a utilização de diversas funções não lineares e filtros digitais para replicar o timbre do aparelho analógico a ser modelado [39]. Geralmente, as transferências não lineares são estáticas (sem memorização). Apesar de não corresponder às características físicas de circuitos reais, essa abordagem pode ser considerada como perceptualmente satisfatória, sendo utilizada em simuladores comerciais de amplificadores [32]. O uso de funções não lineares estáticas tem o propósito de reduzir o esforço computacional e, consequentemente, viabilizar a simulação em tempo real.

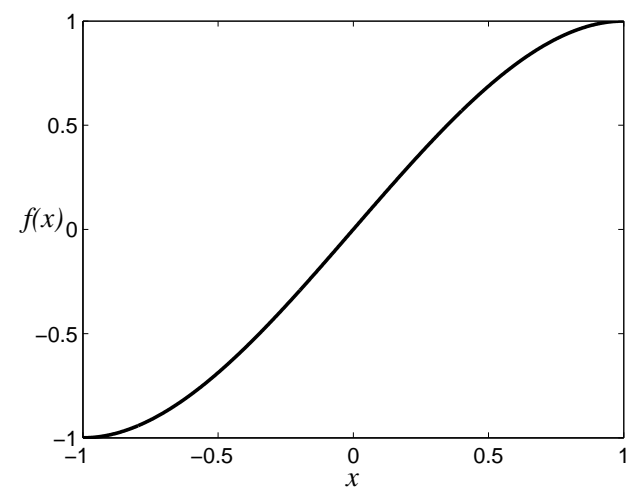

Figura 4: Waveshaper proposto por Araya e Suyama [1]

O waveshaping estático é o método mais simples de se obter uma distorção não linear, sendo considerado uma técnica clássica de síntese sonora digital [2], [20]. Para um waveshaper qualquer, $X$ é o conjunto contendo as amostras a serem processadas, as quais podem ser extraídas do sinal digitalizado do instrumento musical, $x_{n}$ é o valor da $n$-ésima amostra, sendo $x_{n} \in X$, e $f(x)$ a saída da função de waveshaping.

Uma patente de Araya e Suyama [1] para a Yamaha Corporation descreve um dispositivo de efeitos para guitarra que utiliza essa técnica, na qual a distorção valvulada é simulada por meio da função não linear (waveshaper) mostrada na Eq. 1 e ilustrada na Figura 4.

$$
f(x)=\frac{3}{2} x\left(1-\frac{x^{2}}{3}\right)
$$

A Figura 5 ilustra um sinal senoidal de $1 \mathrm{kHz}$ distorcido por esse waveshaper nos domínios do tempo e da frequência, onde os coeficientes da série de Fourier (em dB) foram normalizados para uma maior clareza de representação. Conforme o esperado, o uso de uma função não linear resulta na expansão do conteúdo harmônico do sinal de saída. Visto que esse waveshaper produz apenas uma distorção suave, a patente de Araya e Suyama [1] descreve um sistema DSP em que essa função é aplicada três vezes ao sinal com o objetivo de gerar mais distorção.
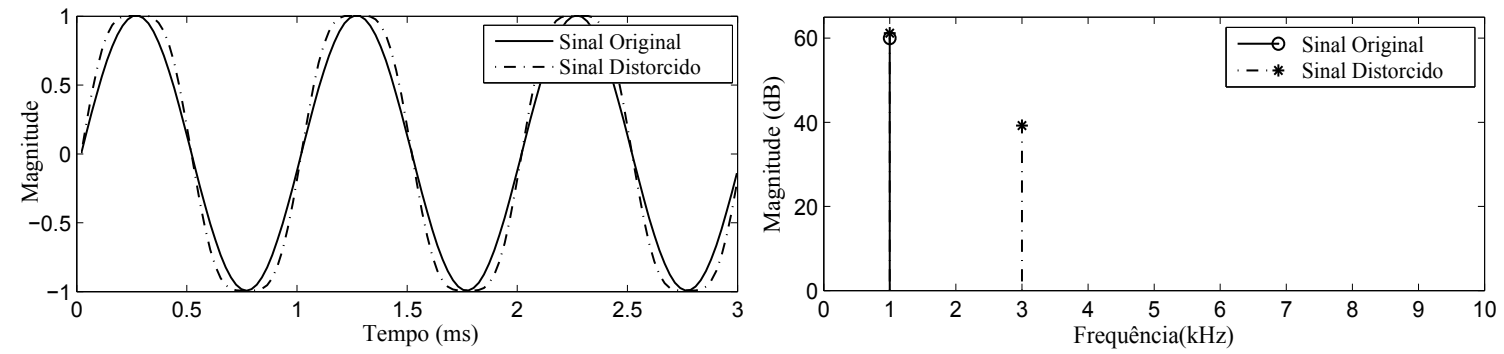

Figura 5: Um sinal senoidal de $1 \mathrm{kHz}$ filtrado pela Eq. 1 no domínio do tempo e da frequência.

A companhia Line 6 [33] patenteou um amplificador digital de guitarra com um simulador de efeitos que 
está registrado sob a patente de Doidic et al. [8]. O diagrama de blocos simplificado desse amplificador é mostrado na Figura 6. O bloco de processamento de sinais digitais é responsável pela geração das diversas distorções não lineares, que são obtidas com os waveshapers estáticos para aproximar o comportamento de diversos amplificadores valvulados ou distorções mais fortes presentes em dispositivos de estado sólido. Vários amplificadores comercializados pela Line 6 com simuladores de amplificadores de guitarra utilizam essas funções. A Eq. 2 representa o waveshaper responsável pela geração de distorções simétricas, consideradas mais suaves e características de amplificadores valvulados, ao passo que a Eq. 3 é utilizada para gerar distorções assimétricas. É importante salientar que a patente original apresenta alguns erros tipográficos na função de distorção assimétrica, de modo que as curvas dos gráficos originais não correspondem à equação presente na patente. Dessa maneira, a Eq. 3 mostrada neste trabalho é uma versão da equação original da patente corrigida de acordo com Pakarinen e Yeh [32]. A Figura 7 mostra as curvas dos waveshapers simétricos e assimétricos gerados pelas Eqs. 2 e 3, que correspondem aos desenhos originais da patente.

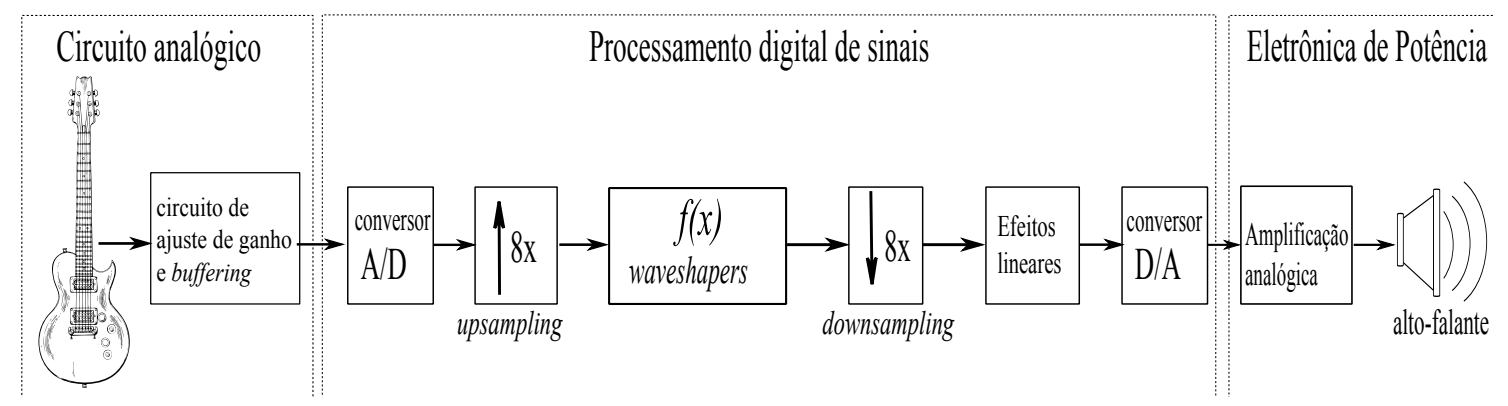

Figura 6: Diagrama de blocos para a simulação de amplificadores valvulados de Doidic et al. [8].

$$
\begin{gathered}
f(x)=\operatorname{sign}(x)\left(2|x|-|x|^{2}\right) \\
f(x)= \begin{cases}-\frac{3}{4}\left\{1-[1-(|x|-0.032847)]^{12}+\frac{1}{3}(|x|-0.032847)\right\}+0.01, & \text { se }-1 \leq x<-0.08905 \\
-6.152 x^{2}+3.9375 x, & \text { se }-0.08905 \leq x<0.320018 \\
0.630035, & \text { se } 0.320018 \leq x \leq 1\end{cases}
\end{gathered}
$$

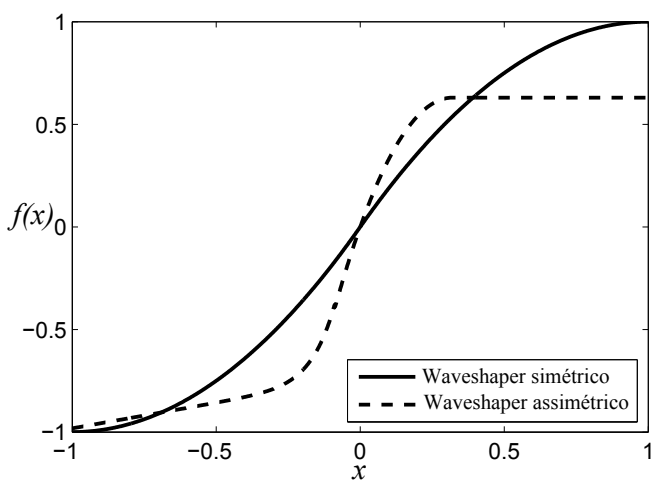

Figura 7: Waveshapers simétricos e assimétricos propostos por Doidic et al. [8].

As Figuras 8 e 9 ilustram as distorções introduzidas pelas Eqs. 2 e 3 em um sinal de teste de $1 \mathrm{kHz}$, respectivamente. Nota-se que a Eq. 3 produz mais distorção do que os demais waveshapers devido à sua assimetria. Por essa mesma razão, esse waveshaper introduz harmônicos pares no espectro do sinal, assim como uma componente DC.

Mais recentemente, Gallo [12] propôs, em uma patente, a Eq. 4 como uma função de waveshaping para simular os efeitos de amplificadores valvulados: 

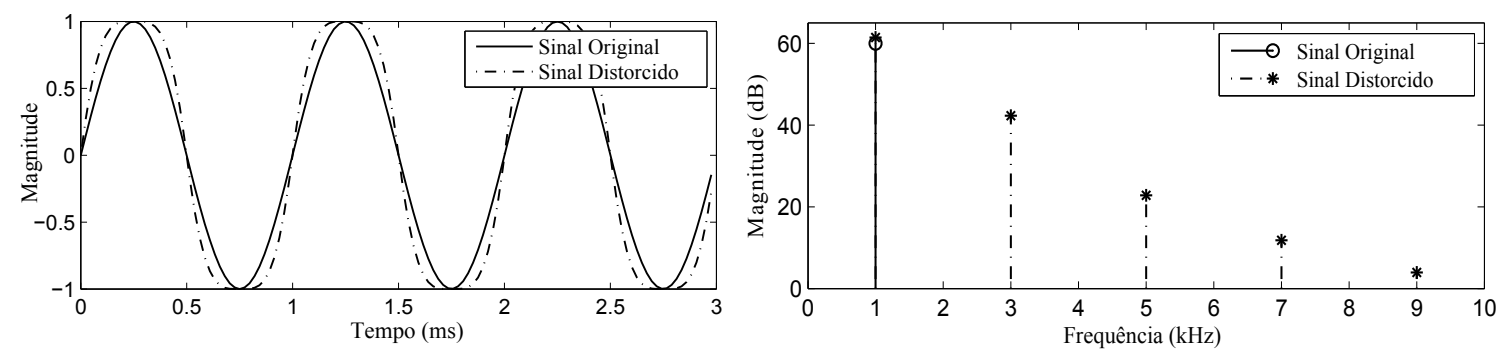

Figura 8: Um sinal senoidal de $1 \mathrm{kHz}$ filtrado pela Eq. 2 nos domínios da frequência e do tempo.
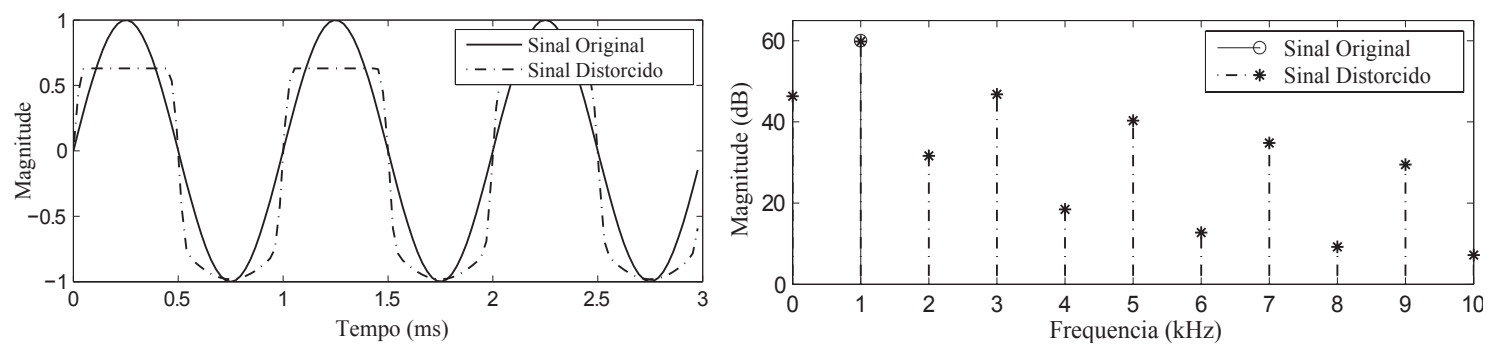

Figura 9: Um sinal senoidal de $1 \mathrm{kHz}$ filtrado pela Eq. 3 nos domínios da frequência e do tempo.

$$
f(x)=\left\{\begin{array}{cll}
\frac{\left(k_{1}+x\right)}{\left(k_{2}-x\right)}, & \text { se } & x<a \\
x, & \text { se } & a \leq x \leq b \\
\frac{\left(x-k_{3}\right)}{\left(x+k_{4}\right)}, & \text { se } & x>b
\end{array}\right.
$$

onde $k_{1}=a^{2}, k_{2}=1+2 a, k_{3}=b^{2}$ e $k_{4}=1-2 b$. Os valores de $a$ e $b$ controlam as características da função não linear e podem ser arbitrados entre $-1.0 \mathrm{e}+1.0$. Uma vez que esses dois parâmetros são independentes um do outro, os valores positivos e negativos do sinal de entrada são tratados separadamente. Isso é desejável para simular o comportamento dos amplificadores valvulados. Sinais de pequena amplitude, entre $a \leq x \leq b$ permanecem sem distorção. A patente de Gallo [12] também descreve outros parâmetros que podem ser adicionados a essa função com o intuito de aumentar a versatilidade sonora do sistema, mas que, por motivos de clareza, foram omitidos deste trabalho.

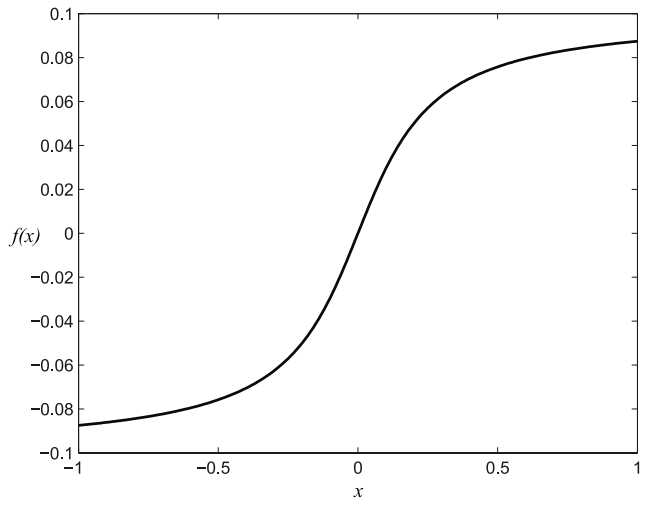

(a)

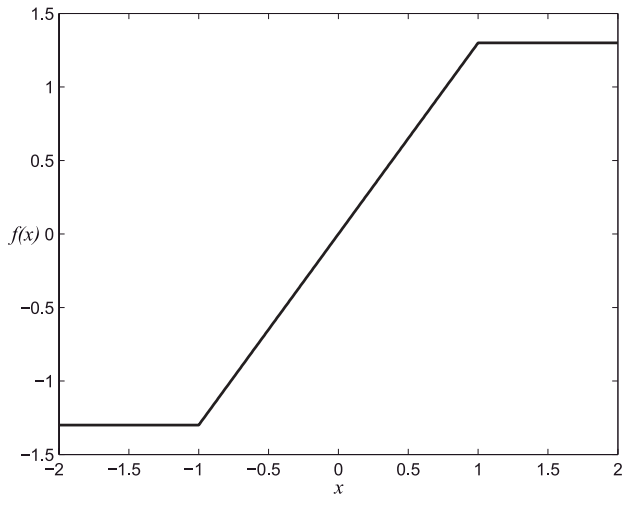

(b)

Figura 10: Função de Waveshaping para a simulação de um pedal de fuzz, (a) Eq. 5 e (b) Eq. 6.

Outro trabalho que utilizou um waveshaper estático foi realizado por Werneck [37], que buscou simular o efeito de distorção de guitarras fuzz do pedal Fuzz Face ilustrado na Figura 1-a. Esse pedal trabalha em regime de voltage feedback com a utilização de dois transistores de Germânio fabricado nos anos 1960. Para a simulação 
desse pedal, utilizou-se um waveshaper por meio de uma equação trigonométrica na forma da Eq. 5. Uma curva típica desse waveshaper pode ser visualizada na Figura 10-a.

$$
f(x)=\frac{\operatorname{atan}\left(k_{d} x\right)}{k_{d} \pi}
$$

A Fig. 11 mostra a distorção introduzida pela Eq. 5 nos domínios do tempo e da frequência para um sinal senoidal de $1 \mathrm{kHz}$ e $k_{d}=3$.
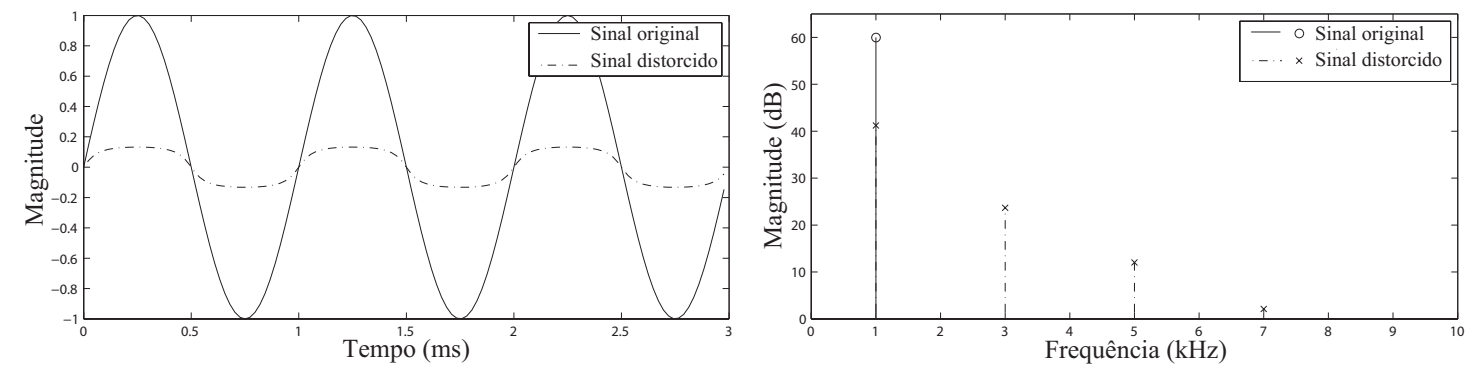

Figura 11: Filtragem de um sinal senoidal de $1 \mathrm{kHz}$ pela Eq. 5 nos domínios do tempo e da frequência.

A Eq. 6, utilizada também na patente de Doidic et al. [8], representa um waveshaper com distorção abrupta de hardclipping análogo aos efeitos de fuzz e de distorções mais fortes, essa mesma função está igualmente inclusa na patente de Araya e Suyama [1]. A Eq. 6 é representada graficamente na Fig. 10-b. Além disso, a distorção gerada pela função de hardclipping num sinal senoidal de $1 \mathrm{kHz}$ com $K=1.3$ é mostrada na Figura 12 nos domínios do tempo e da frequência.

$$
f(x)= \begin{cases}K x & \text { se }|x|<1 \\ K \operatorname{sign}(x) & \text { se }|x| \geq 1\end{cases}
$$
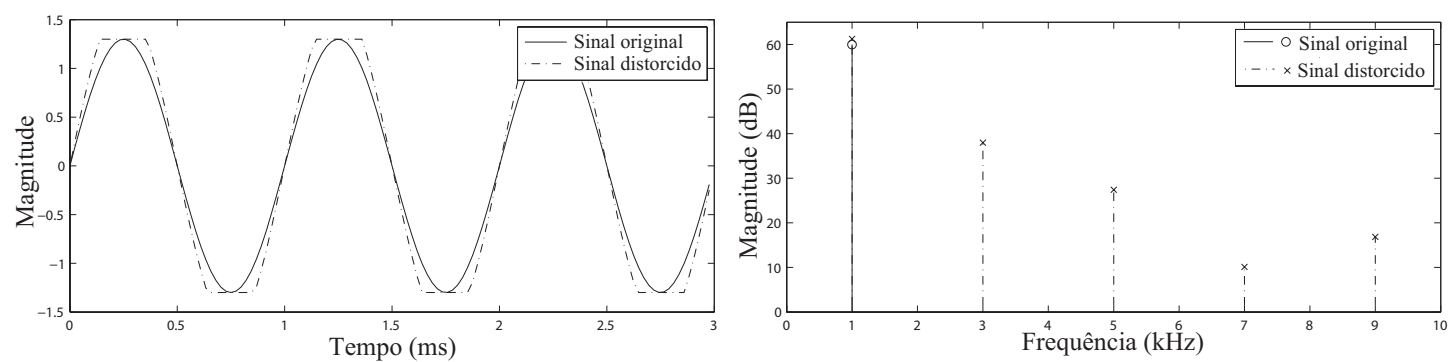

Figura 12: Sinal senoidal de 1 kHz filtrado pela Eq. 6 nos domínios do tempo e da frequência.

\section{Waveshapers originados de circuitos}

Os waveshapers estáticos apresentados na seção 4 foram desenvolvidos sem incluir os fenômenos físicos presentes em circuitos analógicos. Mediante uma abordagem diferenciada, Bendiksen [3] utilizou dados de circuitos na obtenção de um waveshaper estático para simular as distorções de triodos (válvulas com três eletrodos), como é mostrado na Eq. 7:

$$
f(x)=\frac{x-Q}{1-e^{-\operatorname{dist}(x-Q)}}+\frac{Q}{1-e^{\operatorname{dist}(Q)}}
$$


onde $x$ é o sinal de entrada, dist é um parâmetro de ajuste de intensidade da distorção, $Q$ é o ponto de trabalho do triodo e $f(x)$ é o sinal de saída. $Q$ é um parâmetro de todo circuito amplificador que, nesse caso, para um triodo, corresponde à tensão de polarização da grade em relação ao catodo. À medida que $Q$ é aumentado, o sistema aproxima-se da saturação da corrente de placa, e, consequentemente, o sinal de saída é mais distorcido. Por outro lado, quando $Q$ assume valores mais negativos, o sistema apresenta um comportamento aproximadamente linear.

Tipicamente, esse waveshaper gera distorções assimétricas, que são encontradas em amplificadores de triodos quando trabalham em regimes de saturação ou overdrive. Por outro lado, o sinal de saída não será distorcido caso o sinal de entrada seja de baixa amplitude, uma vez que $f^{\prime}(x) \approx 1$. Bendiksen [3] também propõe o uso de um filtro passa-baixas para simular a capacitância de Miller, bem como um filtro passa-altas para atenuar a componente DC introduzida pelo waveshaper assimétrico.

A Eq. 7 irá resultar em diferentes respostas dependendo dos parâmetros aplicados ao waveshaper. Dessa maneira, para fins ilustrativos, a Fig. 13 mostra um sinal senoidal de $1 \mathrm{kHz}$ filtrado pela Eq. 7 com $Q=-1.1 \mathrm{e}$ dist $=1.0$. Antes do processo de waveshaping, um ganho de 10 foi aplicado ao sinal de entrada. Como esperado, a Fig. 13 revela que o espectro do sinal de saída contém harmônicos pares e ímpares, assim como uma componente DC.
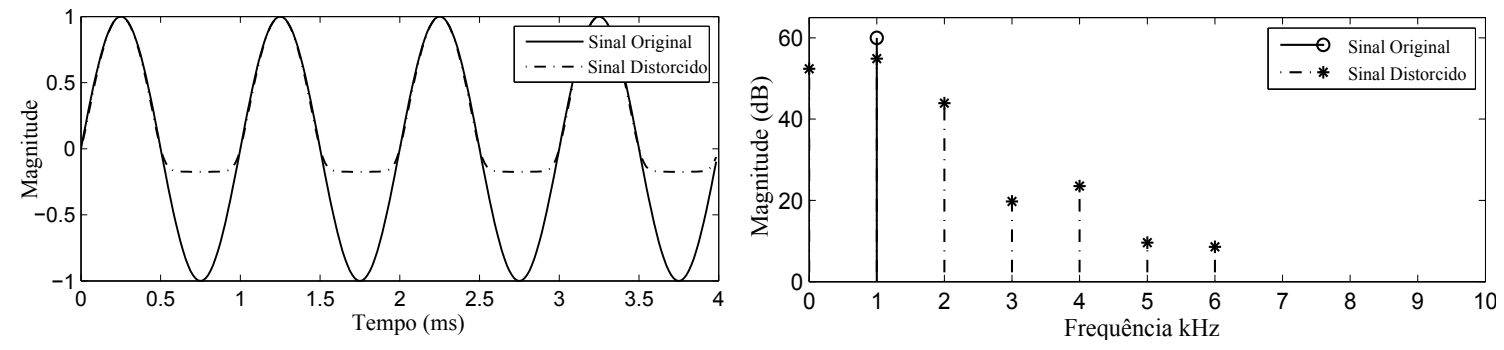

Figura 13: Sinal senoidal filtrado pela Eq. 7 nos domínios do tempo e da frequência.

\section{Aliasing e upsampling}

Sabe-se que a distorção não linear expande a largura de banda de um sinal, conforme evidenciado pelas Figuras 5, 8, 9 e 12. Nos dispositivos distorcedores analógicos, os novos harmônicos são determinados pelo comportamento não linear e a resposta em frequência dos filtros dos circuitos amplificadores. Nos modelos digitais desses dispositivos, a expansão da largura de banda terá efeitos indesejáveis se o teorema da amostragem de Nyquist for violado. Tal teorema impõe que, para realizar a amostragem de um sinal contínuo no tempo de maneira apropriada, a sua frequência máxima deve ser menor do que a metade da frequência de amostragem [25]. Caso contrário, a distorção por aliasing ocorrerá e, como consequência, os harmônicos introduzidos com frequências superiores à máxima permitida irão se desdobrar de volta à largura de banda do sinal como frequências audíveis, gerando efeitos indesejados na simulação da distorção analógica. Nos casos de distorções não lineares mais fortes, isso é mais crítico, uma vez que a largura de banda é expandida de um modo mais pronunciado.

Esse efeito pode ser superado por meio de um incremento da taxa de amostragem do sinal antes do bloco de processamento não linear. Essa é uma técnica amplamente utilizada, sendo chamada de sobreamostragem ou "upsampling” [25]. Como consequência, uma regra de interpolação deve ser empregada para criar valores amostrais entre amostras adjacentes. Claramente, a técnica de upsampling aumenta o tempo de CPU para o processamento não linear. Por essa razão, sistemas de baixo custo para simulação de distorções analógicas utilizam taxas de amostragem mais baixas para o bloco de processamento não linear [32]. A Figura 6 mostra um diagrama de blocos para o sistema descrito na patente de Doidic et al. [8], que ilustra o uso de upsampling para a simulação digital de distorções valvuladas. A taxa de amostragem do sinal discreto no tempo é aumentada por um fator de 8 antes do bloco de processamento não linear. Após o waveshaping, o sinal é filtrado digitalmente por um filtro passa-baixas (com um filtro digital antialiasing, não mostrado na Figura 6), com o objetivo de limitar a largura de banda do sinal. Então, a taxa de amostragem é reduzida com o downsampling para a taxa original, antes de aplicar os efeitos lineares e a conversão D/A. 


\section{Simulação por meio de equações de estados}

A representação de equações no espaço de estados tem sido utilizada para a simulação de efeitos eletrônicos analógicos e tem demonstrado potencial para uma simulação de precisão de diversos circuitos não lineares. Normalmente, junto com essa abordagem, é utilizado um método numérico para a resolução das Equações Diferenciais Ordinárias (EDO), como os métodos de Newton-Raphson, Euler ou Runge-Kutta. Esss modelos são constituídos pelas leis físicas das correntes e tensões de Kirchhoff. As transferências não lineares inseridas nesses sistemas, geralmente, são realizadas por meio dos elementos de circuitos com essas características, como transistores, válvulas ou diodos. Nessa abordagem, utilizam-se equações da literatura que modelam matematicamente esses dispositivos. Os modelos não lineares são inseridos em um sistema de equações que inclui os elementos de circuitos mais simples, como capacitores, resistores e indutores, que compõem o circuito. Esses elementos lineares podem ser modelados matematicamente por equações diferenciais e podem ser convertidos para uma representação no espaço de estados. Por meio de métodos numéricos, o sistema completo pode ser resolvido de maneira iterativa em tempo real [41].

Com a modelagem no espaço de estados, Yeh et al. [42] simulou dois pedais de distorção: o DS-1 da Boss e o Ibanez Tubescreamer. Os mesmos autores, em um outro trabalho [41], realizaram uma comparação entre os métodos de resolução das EDO para estabilidade numérica, precisão, complexidade e outros aspectos para a resolução de sistemas de equações diferenciais ordinárias não lineares. Concluíram que, em geral, métodos como o backward Euller ou a regra trapezoidal são necessários para evitar instabilidade numérica em taxas de amostragem tipicamente utilizadas para a faixa de áudio.

Para a simulação do pedal de alta saturação DS-1, as transferências não lineares do circuito são inseridas por diodos grampeadores em antiparalelo, que grampeiam os picos da onda quando estão conduzindo acima do limiar de 0,7V. Esse tipo de distorção é muito utilizado em pedais e pré-amplificadores e pode ser modelado matematicamente pela Eq. 8 para cada diodo. Uma simulação de um circuito semelhante, da etapa de distorção do amplificador de alto ganho Marshall JCM 900, que utiliza quatro LEDs para ceifar o sinal da guitarra, foi realizada por Dempwolf et al. [7], também fazendo uso de formulações no espaço de estados.

$$
I_{d}(V)=2 I_{s} \sinh \left(V / V_{t}\right)
$$

onde $I_{d}$ é a corrente que circula no diodo, $V$ é a tensão sobre o diodo e $V_{t}$ é um parâmetro de voltagem térmica.

Na simulação do pedal Ibanez Tubescreamer, por Yeh [43], o estágio transistorizado foi modelado segundo os modelos de Ebers-Moll [24] para transistores bipolares. A corrente de emissor pode ser obtida pela Eq. 9, a corrente de coletor pela Eq. 10, e a corrente de base pela Eq. 11.

$$
\begin{gathered}
I_{e}=I_{S} / \alpha_{F}\left[\exp \left(V_{b e} / V_{T}\right)-1\right]-I_{S}\left[\exp \left(V_{b c} / V_{T}\right)-1\right] \\
I_{c}=I_{S}\left[\exp \left(V_{b e} / V_{T}\right)-1\right]-I_{S} / \alpha_{R}\left[\exp \left(V_{b c} / V_{T}\right)-1\right] \\
I_{e}=I_{S} / \beta_{F}\left[\exp \left(V_{b e} / V_{T}\right)-1\right]-I_{S} / \beta_{R}\left[\exp \left(V_{b c} V_{T}\right)-1\right]
\end{gathered}
$$

onde $I_{s}$ é a corrente de saturação, $V_{b e}$ é a tensão base-emissor e $V_{T}$ é um parâmetro de variação de tensão em função da temperatura. As simulações desses dois pedais de distorção por meio de equações de estados foram comparadas com simulações mais simples pelas aproximações estáticas, em [42] e [40], computadas por equações dos dispositivos e posteriormente armazenadas em tabelas para consultas, onde o resultado das aproximações foram considerados bons, sendo computacionalmente mais eficientes para a simulação em tempo real.

No trabalho de Yeh et al. [43], a abordagem é estendida para um estágio de amplificador à válvula triodo (12ax7, 12at7, 6at6, 6av6 etc) pelo modelo de Koren [19] para triodos (Eq. 12 e Eq. 13), sendo os coeficientes das equações omitidos por motivos de clareza neste trabalho. A transformação do sistema de EDO na representação do espaço de estados utilizou o método K, que por meio de métodos implícitos transformam as equações diferenciais ordinais de circuitos em estruturas recursivas no espaço de estados. Essa abordagem utiliza a não linearidade presente no circuito, capacitores de acoplamento e capacitâncias de Miller presentes na própria válvula, possibilitando a modelagem de diversos fenômenos incluídos nesse circuito que não podem ser simulados por waveshapers 
estáticos. A abordagem pode ser considerada uma simulação de força bruta para uma taxa de amostragem fixa (sem sobreamostragem).

$$
\begin{gathered}
E_{1}=\left(E_{p} / k P\right) \log \left(1+\exp \left(k_{p}\left(1 / \mu+E_{g} / \sqrt{k_{V B}+E_{p}^{2}}\right)\right)\right) \\
I_{P}=\left(E_{1} X / k_{G 1}\right)\left(1+\operatorname{sign}\left(E_{1}\right)\right)
\end{gathered}
$$

Com o objetivo de gerar pluggins a partir de esquemas digitalizados de circuitos, Yeh et al. [41] propuseram um método para tal finalidade. Os dados dos circuitos a serem modelados são obtidos por meio de netlists do ambiente Simulation Program with Integrated Circuit Emphasis - (SPICE), que correspondem às malhas dos circuitos propriamente ditas para serem simuladas nesse ambiente. Com essa metodologia, é possível transformar algumas topologias de circuitos simples, como pré-amplificadores de apenas um transistor ou válvula em pluggins de áudio, no sistema operacional LINUX. Os aspectos teóricos desta metodologia são abordados em [41], e os resultados experimentais estão demonstrados em [38], com sucesso para circuitos simples. No entanto, não existem resultados para circuitos mais complexos, como amplificadores de duas ou mais válvulas ou transistores.

Amplificadores valvulados de alto ganho, como o Mesa-Boogie Dual Rectifier e o Marshall JCM800, utilizam muitas válvulas triodo em cascata para maior ganho na distorção. A simulação desses circuitos com mais estágios de amplificação resulta, consequentemente, em desafios técnicos. Macak e Schimmel [21] implementaram a simulação de um Marshall JCM800, um Mesa Boogie Dual Rectifier e um Super Reverb da Fender utilizando equações de Koren [19] de triodos e pentodos. Essas últimas são válvulas com cinco eletrodos, normalmente utilizadas nas etapas de potência dos amplificadores. As interações entre os estágios foram modeladas devido ao fato de a impedância de grade de um estágio subsequente alterar a impedância AC do circuito da placa do estágio anterior. O circuito foi modelado como um sistema não linear de EDO na apresentação do espaço de estados, com resolução numérica pelo método de Newton-Raphson. Outro circuito simulado foi a etapa de potência com pentodos em push-pull, incluindo o circuito da válvula inversora de fase, porém com a ausência do transformador de saída, substituído por um resistor de carga $R_{a}$. Todas as equações da etapa de potência utilizam as equações de pentodo de Koren [19]. Um modelo aprimorado do Marshall JCM800 foi realizado por Mack e Schimmel [22], onde os resultados das equações foram armazenados em uma matriz 3D pré-computada para uma maior eficiência para a utilização do software na forma de pluggins em tempo real. Diferentes métodos de interpolação foram testados para aproximar os valores que não estão presentes na tabela, onde o método de aproximação spline foi considerado o melhor.

Cohen e Helié [5] simularam um amplificador de potência polarizado em classe A com topologia single ended, com um transformador de aúdio por meio das equações de Koren [19] para pentodos (válvulas de cinco eletrodos). Esse trabalho também utilizou a representação pelo espaço de estados para as EDO. Alguns dos parâmetros do transformador foram obtidos com a folha de dados do transformador de áudio Plitron PAT-3050-SE-02, que, nesse caso, foi modelado como um dispositivo linear, apesar do fato de transformadores possuírem efeitos não lineares como saturação do núcleo de ferrite e histerese. O método numérico implícito de Newton-Raphson foi empregado para resolver o sistema de equações não lineares para esse circuito.

\subsection{Equações de Cohen}

Devido ao fato de a emulação digital de circuitos analógicos utilizando sistemas de equações ter uma dependência direta nos modelos dos dispositivos não lineares, é extremamente necessário que esses protótipos aproximem o dispositivo físico de maneira precisa. Como consequência, é desejável que novos modelos surjam com o objetivo de evoluir as simulações. Devido ao fato de os modelos de válvulas termiônicas serem relativamente recentes, sendo o primeiro de Reynalds [34], em 1993, os modelos matemáticos de válvulas termiônicas não atingiram a mesma maturidade de modelos de estado sólido como o de transistores bipolares de Ebers-Moll [24]. O modelo que tem sido amplamente utilizado para a simulação de amplificadores valvulados é o modelo de pentodos e triodos de Norman Koren [19] de 1996. No ano de 2012, Cohen e Helié [6], propuseram novos modelos de válvulas triodos, os quais foram obtidos experimentalmente com triodos reais e comparados às equações de Koren. As novas equações utilizam parâmetros cujo objetivo é corrigir o modelo de Koren em regiões de baixa tensão de placa e tensões positivas entre a grade e o catodo. Outro aspecto importante desse modelo é o fato de a corrente de grade ser incluída nesse protótipo. Os modelos anteriores descartam a corrente de grade, admitindo que a impedância da malha grade-catodo possui impedância infinita. A inclusão de uma corrente não nula para o circuito 
da grade torna o modelo mais completo e teoricamente mais próximo da realidade física do triodo. Por outro lado, o fato de Cohen não ter proposto um novo modelo fenomenológico para os pentodos, leva a que desenvolvedores de softwares emuladores de amplificadores valvulados que incluam a etapa potência ainda façam uso dos modelos de Koren para pentodos.

\section{Wave Digital Filters}

Os Wave Digital Filters, ou apenas WDF, constituem uma metodologia utilizada para modelar amplificadores valvulados fisicamente entre outros circuitos, bem como para gerar modelos físicos de instrumentos musicais por meio de modelos mecânicos [15]. Originalmente, os WDF foram desenvolvidos com o propósito de digitalizar circuitos complexos com muitos componentes. Um desenvolvimento aprofundado acerca do assunto pode ser encontrado no trabalho de Alfred Fetwis [10].

Os WDF são um tipo de filtro digital que possuem uma interpretação válida no mundo real. Isso significa que podemos simular o comportamento de um sistema físico agregado e complexo por meio de coeficientes que têm a sua dependência nos parâmetros desse sistema físico [36].

Ao contrário da maioria dos modelos digitais de amplificadores e efeitos de distorção, em que o sinal é tratado com filtros lineares e waveshapers não lineares, possivelmente com parâmetros que possuem dependência no sinal, sendo o fluxo do sinal do modelo unidirecional, um modelo WDF possui um comportamento bidirecional entre dois ou mais componentes em um circuito, como exemplificado na Figura 14.

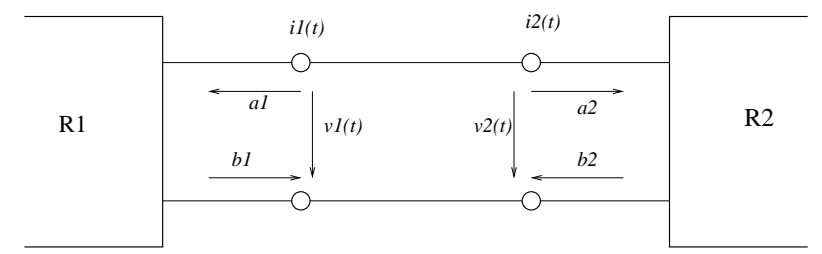

Figura 14: Interconexão de dois dispositivos de duas portas.

Com a interconexão bidirecional entre componentes, torna-se possível a realização de modelos complexos como de um pré-amplificador com válvula 12AX7, como o da Figura 15, onde (a) representa o esquema do circuito clássico de amplificação de catodo comum e (b) corresponde à representação WDF desse circuito. Um modelo WDF é convertido em código C-Lisp para a simulação no software Block Compiler desenvolvido pelo pesquisador Matti Karjalainen [14]. O software é, basicamente, um tradutor de linguagem C-lisp para código em liguagem em $\mathrm{C}$ ou Matlab. Sendo uma liguagem do paradigma funcional, o C-Lisp permite uma complexa descrição de objetos por meio de arranjos de listas ou listas recursivas. Devido à complexidade de estruturas que podem ser detalhadas, uma poderosa descrição de malhas de circuitos e sistemas complexos pode ser implementada [17].

\subsection{Modelos de triodos em Wave Digital Filters}

Alguns modelos WDF de circuitos valvulados de triodos foram implementados. A modelagem matemática de triodos é feita por meio de um resistor não linear e das equações de Koren [19], onde a resistência de placa do triodo é controlada pela tensão de grade de maneira análoga ao modo como a corrente de placa é controlada pela tensão de grade nessas válvulas.

Em [18], a metodologia é utilizada para modelar um estágio de amplificação com um triodo 12ax7, sendo esse um estágio presente na maioria dos amplificadores valvulados de guitarra na parte de pré-amplificação. A Figura 15 (b) ilustra o diagrama desse modelo, que utiliza as equações de Koren [19], 12 e 13, como modelos não lineares para triodos.

As equações foram computadas em tempo de compilação (tabela pré-computada) e utilizadas para consulta do mapeamento $f\left(V_{g k}, V_{p k}\right) \rightarrow I_{p}$, durante a execução da simulação, para ganho em desempenho.

Um aprimoramento do modelo de triodos foi realizado por Pakarinen et al. [30]. Esse trabalho possui, na 


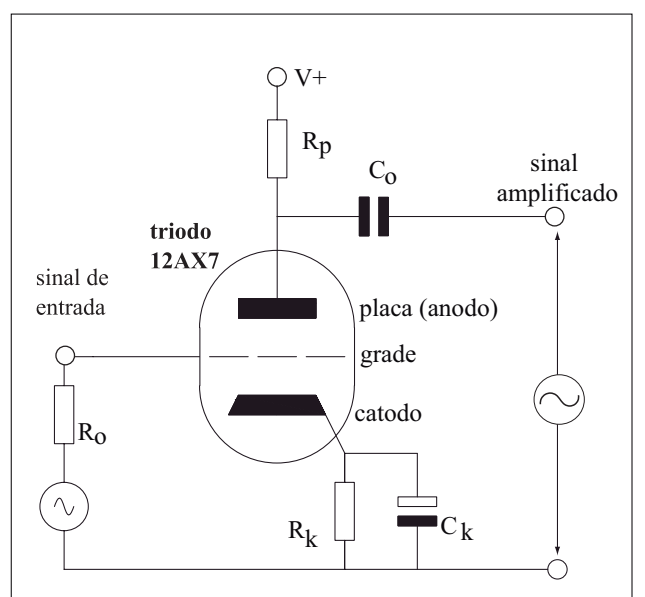

(a)

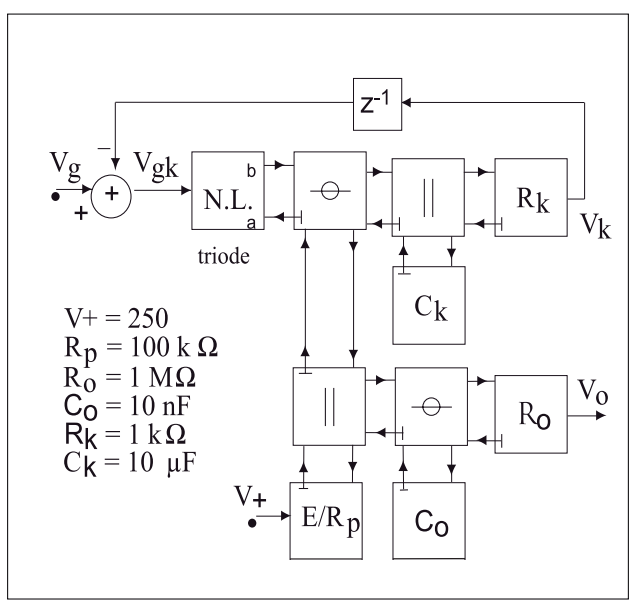

(b)

Figura 15: Pré-amplificador em configuração catodo comum com uma 12AX7.

modelagem, os efeitos de capacitância de Miller e de distorção blocante. Os efeitos reativos de capacitância entre os circuitos também foram modelados. Contudo, esse modelo obteve um resultado quase idêntico à simulação no ambiente SPICE e pode ser implementado em tempo real pelo Block Compiler, por meio de um tradutor de código C-Lisp para C com o ambiente MinGW.

Um modelo WDF de toda a cadeia do sinal com a etapa de saída de um amplificador também foi implementado por Pakarinen [31], desde o primeiro estágio a triodo até a etapa de potência, que foi simulada por modelos de triodos com equações de Koren [19]. O triodo modelado foi a válvula KT88 operando como triodo em topologia single ended, ao contrário da maioria dos amplificadores, que utiliza pentodos de alta potência como a válvula KT88 operando como pentodo. Outro aspecto notável é que a válvula de saída é polarizada em classe single ended, encontrada apenas em amplificadores de baixa potência e circuitos de alta fidelidade. A maioria dos amplificadores utiliza pentodos em configuração push-pull polarizados em classe AB.

Os transformadores de áudio que são inseridos na cadeia de amplificação do sinal entre o alto-falante e as válvulas de potência têm a função de realizar o casamento de impedâncias entre as válvulas (alta impedância na ordem de $k \Omega$ ) e os alto-falantes que possuem a impedância tipicamente entre $4 \Omega$ e $16 \Omega$. É conhecido o fato de esse tipo de transformador inserir distorções não lineares no sinal, por meio da saturação e histerese do núcleo. Um modelo computacional WDF com esses fenômenos do transformador foi proposto por Paiva et al. [26] e implementado utilizando-se de dados obtidos experimentalmente. As equações desse modelo foram baseadas em um protótipo de transformador Girator-Capacitor, que inclui os efeitos de histerese e de saturação do núcleo de ferrite.

Após o falecimento de Matti Karjalainen (1946-2010), o idealizador e programador do Block Complier, o projeto foi abandonado e o suporte ao software foi interrompido. A última versão é considerada instável para implementar modelos físicos mais complexos. $\mathrm{Na}$ atualidade, pesquisadores estão trabalhando em ferramentas que têm o potencial de substituír o Block Compiler, uma vez que a abordagem WDF é considerada promissora para a simulação de sistemas físicos complexos como circuitos de distorção não lineares. O ambiente CSound demonstrou potencial para a abordagem WDF para circuitos de distorção, conforme um trabalho de Fink et al. [11], no qual o clássico circuito de triodo em configuração catodo-comum da Figura 15 foi simulado em tempo real através de códigos de operações Csound.

\section{Conclusões}

Devido à facilidade de difusão, os softwares de simulação de distorções analógicas estão sempre atraindo novos usuários. Diversos modelos têm sido propostos, com vários graus de complexidade e precisão, no entanto, ainda não são capazes de reproduzir satisfatoriamente a qualidade das distorções analógicas, principalmente as 
geradas por equipamentos valvulados. Os métodos baseados na simulação dos circuitos são os mais utilizados na atualidade e os que apresentam os melhores resultados. Contudo, para a simulação em tempo real, deve-se levar em conta o compromisso entre precisão e tempo computacional. Além disso, mais pesquisas devem ser realizadas com o objetivo de gerar um novo conjunto de equações que descreva satisfatoriamente os fenômenos físicos das válvulas, pois as equações de Cohen [6] para triodo são relativamente recentes, sem precisão comprovada. Uma sumarização comparativa entre alguns dos métodos foi feita na Tabela 1, onde a complexidade assintótica do método é inserida, assim como um resumo da qualidade da simulação. A complexidade está em função do número de nós no circuito, ou seja, em waveshapers, a complexidade é considerada constante devido a esses algoritmos apenas aproximarem o comportamento de circuitos mais complexos. Nessa tabela, salienta-se que os métodos mais simples sempre têm a complexidade constante, em razão de o número de operações ser sempre o mesmo para cada amostra do sinal. À medida que a complexidade dos métodos aumenta, estes demandam maior recurso computacional. Em razão de as simulações presentes na literatura abordarem circuitos com diferentes topologias, a sumarização comparativa baseia-se nos dados originados dos trabalhos referenciados, e a comparação entre os métodos, nessa tabela, serve como guia para o entendimento sobre cada um deles. Outro aspecto importante é que o SPICE é considerado o melhor método de simulação, sendo utilizado para validação dos modelos "analogicamente virtuais", porém, a sua alta complexidade computacional inviabiliza o seu uso em tempo real, pois são gastos vários segundos para a simulação de $1 \mathrm{~ms}$ de um circuito de um triodo apenas. É importante ressaltar que a comparação entre as distintas formas de onda distorcidas em diferentes algoritmos de processamentos de sinais é difícil, sendo o ouvido treinado de um bom músico ainda considerado a melhor ferramenta para o julgamento entre diferentes métodos [32].

\begin{tabular}{|c|c|c|}
\hline Método & Complexidade & Precisão do método \\
\hline Waveshaper estático & $\mathrm{O}(\mathrm{n})=\mathrm{C}$ & Pouca precisão \\
Waveshaper dinâmico & $\mathrm{O}(\mathrm{n})=\mathrm{C}$ & Mais preciso que o estático \\
Wave Digital Filters [27] & $\mathrm{O}(\mathrm{n})=\mathrm{n}$ & Resultados similares ao SPICE \\
Equações de estados [21] & $O(n)=C=5.28 \times 10^{4}$ & Resultados similares ao SPICE \\
Equações de estados (aproximadas) [21] & $O(n)=9.53 \times 10^{2}$ & Resultados similares ao SPICE \\
SPICE & $O(n)=N^{1.4}$ & Método mais preciso de todos \\
\hline
\end{tabular}

Tabela 1: Sumarização comparativa entre métodos de simulação de distorções para guitarras elétricas

No contexto discutido anteriormente, salienta-se que, devido à alta complexidade dos sistemas não lineares discutidos neste trabalho, a análise da qualidade dos emuladores de circuitos amplificadores é extremamente difícil. Finalmente, é importante frisar que há uma lacuna no que tange a comparações subjetivas entre dispositivos distorcedores virtuais e reais, como o teste de "Auditory Sharpness" de Marui e Martens [23]. Nesse sentido, a investigação psicoacústica revela-se potencialmente útil na validação dos modelos propostos e poderia ser utilizada como guia para futuros trabalhos nessa área, assim como método comparativo entre diferentes modelos.

\section{Referências}

[1] Araya, T. E Suyama, A. Sound effector capable of imparting plural sound effects like distortion and other effects. U.S. Patent no. 5570424, Outubro 1996.

[2] ARFIB, D. Digital synthesis of complex spectra by means of multiplication of nonlinear distorted sine waves. Journal of the Audio Engineering Society 27, 10 (1979), 757-768.

[3] Bendiksen, R. Digitale lydeffekter. M.sc thesis, Norwegian University of Science and Technology, Agosto 1997.

[4] Bussey, W. E Haigler, R. Tubes versus transistors in electric guitar amplifiers. In Acoustics, Speech, and Signal Processing, IEEE International Conference on ICASSP 1981 (Atlanta, Georgia, E.U.A., Abril 1981), pp. $800-803$.

[5] Cohen, I. E Helié, T. Measures and parameter estimation of triodes, for the real-time simulation of a multistage guitar preamplifier. In Proceedings of the 109th AES Convention (San Francisco, California, E.U.A., Fevereiro 2000). 
[6] Cohen, I. E HÉlie, T. Measures and models of real triodes, for the simulation of guitar amplifiers. In Proceedingseedings of the Acoustics 2012 Nantes Conference (Nantes, França, Abril 2012).

[7] Dempwolf, K., Holters, M., e Zölzer, U. Discretization of parametric analog circuits for real-time simulations. In Proceedings of the 13th International Conference on Digital Audio Effects (DAFx-10) (Graz, Áustria, Setembro 2010).

[8] Doidic, M., Ryle, M. E Senffner, C. Tube modeling programmable digital guitar amplification system. U. S. Patent no 20040258250, Agosto 1998.

[9] DutilleuX, P. E Zölzer, U. DAFX - Digital Audio Effects. John Wiley \& Sons, Ltd, Publication, Brandenburgh, Alemanha, 2010, ch.5 Nonlinear Processing, pp. 93-136.

[10] FetTweis, A. Wave digital filters: Theory and practice. Proceedings of the IEEE 74, 2 (1986), $270-327$.

[11] Fink, M. E Rabenstein, R. A csound opcode for a triode stage of a vacuum tube amplifier. In Proceedings of the 9th International Conference on Digital Audio Effects (DAFx-11) (Paris, França, 2011, Setembro 2011), pp. 1-6.

[12] Gallo, M. N. Method and aparatus for distortion of audio signals and emulators of vacuum tube amplifiers. U.S. Patent no. 2011/0033057, Fevereiro 2011.

[13] Godsill, S. E Rayner, P. Applications of Digital Audio Signal Processing in Audio And Accustics. Kluer Academic Publishers, Brandenburgh, Alemanha, 1998, ch. 4, pp. 133-193.

[14] Karjalainen, M. Block compiler - a research tool for physical modeling and dsp. In Proceedings of the 6th Int. Conferenceon Digital Audio Effects (DAFx-03) (Londres, Grã-Bretanha, Setembro 2003).

[15] Karjalainen, M. Discrete-time modeling and synthesis of musical instruments. In Joint Baltic-Nordic Acoustics Meeting (Mariehamn, Åland, Finlândia, Junho 2004).

[16] Karjalainen, M. A block-based multi-paradigm approach with applications to audio and accustics. Tecnical report, Helsinki University of Technology · Laboratory of Acoustics and Audio Signal Processing, 2008.

[17] Karjalainen, M. Efficient realization of wave digital components. IEEE Transactions On Audio, Speech, and Language Processing 16, 5 (2008), 738-746.

[18] Karjalainen, M. E Pakarinen, J. Wave digital simulation of a vacuum-tube amplifier. In Acoustics, Speech and Signal Processing, 2006. ICASSP 2006 Proceedings (Toulouse, França, Julho 2006).

[19] Koren, N. Improved vacuum tube models for spice simulations. Glass Audio 8, 5 (1996), 18-27.

[20] Le Brun, M. Digital waveshaping synthesis. Journal of the Audio Engineering Society 27, 4 (1979), 250-266.

[21] MacAK, J. E Schimmel, J. Real-time guitar tube amplifier simulation using approximation of differential equations. In Proceedings. of the 13th International Conference on Digital Audio Effects (DAFx-10) (Graz, Áustria, Setembro 2010).

[22] MACAK, J., AND SCHIMMEL, J. Real-time guitar preamp simulation using modified blockwise method and approximations. EURASIP - Journal on Advances in Signal Processing 2011629309 (2011), 1-11.

[23] Mauri, A. E Martens, W. Timber of non-linear distortion effects: Perceptual atributes beyond sharpness. In Proceedings of the Conference on Interdisciplinar Musicology (CIM05) (Québec, Canada, Março 2004).

[24] Muller, R. S., Kamins, T. L. E Chan, M. Device Electronics for Integrated Circuits. John Wiley \& Sons, Inc., Hoboken, New Jersey, E.U.A., 2002.

[25] Oppenheim, A. V., Schafer, R. W. Discrete-Time Signal Processing, terceira ed. Prentice Hall Signal Processing Series - Pearson Education Ltd., Londres, Grã Bretanha, 2009. 
[26] Paiva, R., Pakarinen, J., VÄlimäki, V. E Tikander, M. Real-time audio transformer emulation for virtual tube amplifiers. EURASIP - Journal on Advances in Signal Processing 2011, 347645 (2011), 1-15.

[27] PAKarinen, J. Modeling of nonlinear and time-varying phenomena in the guitar. Ph. d. thesis, Helsinki University of Technology Faculty of Electronics, Communications and Automation Department of Signal Processing and Acoustics, Março 2008.

[28] PAKARINen, J. Distortion analysis toolkit a software tool for easy analysis of nonlinear audio systems. EURASIP - Journal on Advances in Signal Processing 2011, 940784(2011), 1-15.

[29] Pakarinen, J., Abel, J., Fontana, Federico, L. V. E VÄlimäki, V. Musical applications of real-time signal processing. EURASIP - Journal on Advances in Signal Processing 2011, 108 (2011), 1-2.

[30] Pakarinen, J. E Karjalainen, M. Enhanced wave digital triode model for real-time tube amplifier emulation. IEEE Transactions on Audio, Speech, and Language Processing 18, 4 (Maio 2010), 738 -746.

[31] Pakarinen, J., Mirkka, T. E Karjalainen, M. Wave digital model of the output chain of a vacuum-tube amplifier. In Proceedings of the 12th International Conference on Digital Audio Effects (DAFx-09), (Como, Itália, Setembro 2009).

[32] Pakarinen, J. E YeH, D. T. A review of digital techniques for modeling vacuum-tube guitar amplifiers. Computer Music Journal 33, 2 (2009), 85-100.

[33] PERry, T. S. Dave fruehling: His job rocks! IEEE Spectrum 41, 2 (2004), 40-41.

[34] ReYnolds, S. Vacuum-tube models for pspice simulations. Glass Audio 5, 4 (1993), 17-25.

[35] Smith, S. Digital Signal Processing: A Practical Guide for Engineers and Scientists . Newnes, Burlington, Carolina do Norte, E.U.A., 2003.

[36] Välimäki, V., Pakarinen, J., Erkut, C. e Karjalainen, M. Discrete-time modelling of musical instruments. Reports on Progress in Physics 69, 1 (2006), 1-78.

[37] Werneck, N. L. Análise da distorção musical de guitarras elétricas. Tese de mestrado, UNICAMP, Faculdade de Engenharia Elétrica e Computação, Agosto 2007.

[38] YEH, D. T. Automated physical modeling of nonlinear audio circuits for real-time audio effects - part II: Bjt and vacuum tube examples. IEEE Transactions on Audio, Speech, and language Processing 20, 4 (2012), 1207-1216.

[39] Yeh, D. T., Abel, J. E Smith III, J. O. Simplified physically-informed models of distortion and overdrive guitar effects pedals. In Proceedings. of the 10th International Conference on Digital Audio Effects (DAFx07) (Bordeux, França, Setembro 2007).

[40] Yeh, D. T., Abel, J. E Smith III, J. O. Simulation of the diode limiter in guitar distortion circuits by numerial solution of ordinary differential equations. In Proceedings of the 10th International Conference on Digital Audio Effects (DAFx-07) (Bordeux, França, Setembro 2007).

[41] Yeh, D. T., Abel, J. S. e Smith III, J. O. Automated physical modeling of nonlinear audio circuits for real-time audio effects - part I:theoretical development. IEEE Transactions on Audio, Speech, and language Processing 18, 4 (2010), 728-737.

[42] Yeh, D. T., Abel, J. S., Vladimirescu, A., E Smith III, J. O. Numerical methods for simulation of guitar distortion circuits. Computer Music Journal 32, 2 (2008), 23-42.

[43] Yeh, D. T. E SMith III, J. O. Simulation guitar distortion circuits using wave digital and nonlinear statespace formulations. In Proceedings. of the 11th International Conference on Digital Audio Effects (DAFx-07) (Bordeux, França, Setembro 2008). 